\title{
Comparison of matrix metalloproteinase-9 and E-cadherin expression in early- and late-onset preeclampsia
}

\author{
Tjam Diana Samara ${ }^{1,2^{*}}$, Heri Wibowo ${ }^{1,3}$, Isabella Kurnia Liem ${ }^{1,4}$, Ani Retno Prijanti1,5, \\ and Andrijono ${ }^{1,6}$
}

\begin{abstract}
BACKGROUND

Preeclampsia (PE) is one of the most common pregnancy complications worldwide. Turnover of villous trophoblast is affected by impaired placental perfusion in preeclampsia. Among the various factors that influence pro and antiangiogenic factors in trophoblast invasion of PE are E-cadherin and matrix metalloproteinase-9 (MMP-9). The current classification scheme differentiates PE into two variants early-onset (EO) and late-onset (LO) PE. The aim of this study was to compare MMP-9 and E-cadherin expression between early- (EO) and late-onset (LO) PE.
\end{abstract}

\section{METHODS}

This study used a cross-sectional design involving 26 women with gestational age $<34$ weeks (EO) and 38 women with gestational age $\geq 34$ weeks (LO) from PE patients. Placentas born to preeclamptic mothers were taken in the form of small pieces of the maternal side to measure the levels of MMP-9 and E-cadherin by the ELISA method. Statistical analysis was assessed using the Mann Whitney and independent t-test with a significant $\mathrm{p}$ value $<0.05$.

\section{RESULTS}

Semiquantitative proteinuria levels were significantly higher in EO-PE group compared to LO-PE group ( $\mathrm{p}=0.000)$. Mean E-cadherin levels were significant lower in the EO-PE group $(125.94 \pm 54.22 \mathrm{pg} / \mathrm{mg})$ compared to LO-PE group $(157.95 \pm 54.12 \mathrm{pg} / \mathrm{mg})(\mathrm{p}=0.024)$. However, there was no significance difference in median MMP-9 levels between EO-PE group and LO-PE group $(\mathrm{p}=0.376)$.

\section{CONCLUSION}

This study demonstrate that E-cadherin had lower levels in preeclampsia patients who gave birth $<34$ weeks. This study indicated that lower levels of e-cadherin can lead to early delivery in preeclampsia patients.

Keywords: MMP-9, E-cadherin, early-onset preeclampsia, late-onset preeclampsia
${ }^{1}$ Biomedical Science Department, Faculty of Medicine, Universitas Indonesia, Jakarta, Indonesia ${ }^{2}$ Anatomy Department, Faculty of Medicine, Universitas Trisakti, Jakarta, Indonesia

${ }^{3}$ Parasitology Department, Faculty of Medicine, Universitas Indonesia, Jakarta, Indonesia

${ }^{4}$ Anatomy Department, Faculty of Medicine, Universitas Indonesia, Jakarta, Indonesia

${ }^{5}$ Biochemistry and Molecular Biology Department, Faculty of Medicine, Universitas Indonesia, Jakarta, Indonesia

${ }^{6}$ Obstetric and Gynaecologist Department, Faculty of Medicine, Universitas Indonesia, Jakarta, Indonesia

Correspondence :

*Tjam Diana Samara Anatomy Department, Faculty of Medicine, Universitas Trisakti, Jl. Kyai Tapa no.260 Grogol Jakarta Email: dianasamara@trisakti.ac.id ORCID ID: 0000-0003-0610-0638

Date of first submission, May 3, 2021 Date of final revised submission, September 15, 2021

Date of acceptance, September 23, 2021

This open access article is distributed under a Creative Commons AttributionNon Commercial-Share Alike 4.0 International License

Cite this article as: Samara TD, Wibowo H, Liem IK, Prijanti AR, Andrijono. Comparison of matrix metalloproteinase-9 and E-cadherin expression in early- and late-onset preeclampsia. Univ Med 2021;40:200-6. doi: 10.18051/UnivMed.2021.v40.200206 


\section{BACKGROUND}

Preeclampsia (PE) is a clinical syndrome characterized by the onset of hypertension at more than 20 weeks of gestation or immediately after delivery, and is accompanied by proteinuria or thrombocytopenia, kidney failure, liver failure, pulmonary edema, cerebral disorders, or visual disorders. ${ }^{(1)}$ Preeclampsia is a major cause of morbidity and mortality, both in the mother and in the fetus. The worldwide incidence of PE is about $2-8 \%$ of all pregnancies, with maternal mortality ranging from $10 \%-15 \%{ }^{(2)}$ In Indonesia, the maternal mortality rate of 225/100,000 deliveries is still high, where pre-eclampsia and eclampsia as most possible cause..$^{(3)}$ Many factors that can cause PE, such as obesity, family history of PE, maternal age ( $>35$ years or $<17$ years), blood vessel disorders such as chronic kidney disease and diabetes mellitus, hypertension, thrombotic disorders such as antiphospholipid syndrome, multiple pregnancies, nulliparous versus multiparous, and immune maladaptive reaction. ${ }^{(4)}$ In pathogenesis, one of the factors that is considered to be the cause of $\mathrm{PE}$ is due to shallow placentation and deficient remodeling of uterine spiral arteries by invasive placental trophoblast cells which results in impaired perfusion of the fetus-maternal unit. ${ }^{(5)}$ The invasion disruption is caused by an imbalance between proangiogenic and antiangiogenic factors. This imbalance is thought to be due to the antiangiogenic protein appearing too early and/or if the production is excessive. ${ }^{(6)}$ Among the various factors that influence pro and antiangiogenic factors are Ecadherin and matrix metaloproteinase-9 (MMP9). ${ }^{(7,8)}$

E-cadherin is one of the most important molecules in adhesion cells in epithelial tissue. ${ }^{(9)}$ E-cadherin is located on the surface of epithelial cells in areas of contact with cells known as adherent junctions. Suppression of E-cadherin expression is the main cause of dysfunction in cell adhesion. One of the important functions of E-cadherin in development is its control of epithelial-mesenchymal transition. E-cadherin, which is involved in wnt signaling, plays a role in regulating migration, proliferation, apoptosis, and differentiation of cells. ${ }^{(10)}$ During the first trimester of pregnancy, the expression of Ecadherin in trophoblasts temporarily decreases so that trophoblast cells have the potential to migrate and invade. ${ }^{(11)}$

Matrix metaloproteinase-9 is the main mediator for extracellular matrix (ECM) degradation and has a role in placental angiogenesis by transforming of artery structural. ${ }^{(12)}$ Matrix metaloproteinase-9 degrades ECM by activation of major proangiogenic factors such as vascular endothelial growth factor (VEGF) and fibroblast growth factor (FGF2). ${ }^{(13)}$ Matrix metaloproteinase-9 degrades E-cadherin to soluble E-cadherin, which suppresses Ecadherin activity and induces EMT. ${ }^{(14)}$ Matrix metaloproteinase- 9 expression increases from 8 to 11 weeks, becoming the main gelatinase until late in pregnancy. ${ }^{(15)}$ MMP-9 turut berperan dalam invasi sel trofoblas dan ekspresinya banyak didapatkan pada sel stroma desidual. ${ }^{(16)}$ Sosa et al. ${ }^{(15)}$ found that MMP-9 has a major role during invasion and contributes to the remodeling of the uterine spiral artery. This is because MMP as a specific proteolytic enzyme plays an important role in the degradation of the extracellular matrix resulting in invasion of proliferative cells into the surrounding tissue.

The current classification scheme differentiates PE into two variants. ${ }^{(17)}$ Early-onset PE (EO-PE) develops to and requires delivery at 34 weeks of gestation. Late-onset PE (LO-PE) appears after 34 weeks of pregnancy as a metabolic syndrome and is not associated with the formation and functioning of the placenta in the second half of pregnancy.

A study showed that in EO-PE group the MMP-9 expression in placenta tissues was siginificantly decreased compared to normal pregnancy. ${ }^{(18)}$ Another study demonstrated increased expression of E-cadherin in late onset preeclamptic compared to that in gestation normotensive pregnancies. ${ }^{(19)}$ Furthermore, there was no difference in the expression of E-cadherin 
between early onset and late onset preeclampsia. ${ }^{(19)}$

At present, the role of vascular endothelial injury in EO-PE and LO-PE are concerned, but its regulation mechanism is not clear, and the results of previous studies were inconclusive. Therefore, the aim of this study was to compare the MMP-9 and E-cadherin expression between EO-PE and LO-PE patient.

\section{METHODS}

\section{Research design}

This study was a cross-sectional design with analytic observations. Sampling was carried out at Cipto Mangunkusumo Hospital (RSCM) and Budi Kemuliaan Hospital, Jakarta, Indonesia from April 2017 to April 2018.

\section{Research subject}

The sample size was determined based of the results of preliminary study of 5 subjects showed that the mean difference of MMP-9 between EO-PE and LO-PE was 5.6, $\mathrm{SD}=1.66$ (data not shown). Using the comparison between two independent group equation with $\alpha=0.05$ and $\beta=0.2$, the sample size for each group was 38 to done to get sample size. The study was divided into two groups: EO- and LO-PE. Each group needed 38 samples. The samples were collected until the number of samples was met. The subjects were 19 years or more and had agreed to participate in this study by signing an informed consent were included into this study. Patients with renal failure and diabetes mellitus were excluded from this study. The placentas were removed at the time of the patient's delivery either by vaginal or caesarean section.

\section{Measurements}

According to The American College of Obstetrics and Gynaecologists (ACOG), preeclampsia is a clinical syndrome characterized by hypertension that appears after 20 weeks of gestation (systolic pressure $\geq 140 \mathrm{mmHg}$ and/or diastolic pressure $\geq 90 \mathrm{mmHg}$ ), and proteinuria ( $\geq 300 \mathrm{mg}$ in 24 hours urine) or with positive protein $(1+)$ taken from mid-stream urine. Preeclampsia can be without proteinuria but must be accompanied by any of the following symptoms: thrombocytopenia $(<100,000 / \mathrm{mL})$, renal failure (serum creatinine $>1.1 \mathrm{mg} / \mathrm{dL}$ ) abnormal liver function (transaminases are twice as high as normal), pulmonary oedema, and cerebral disorders or visual disorders. ${ }^{(1)}$

\section{Specimen collection}

Maternal placental cotyledons were removed $0.5 \times 0.5 \times 0.5 \mathrm{~cm}$ immediately after vaginal birth or caesarean section. A total of 100 mg of placental tissue was washed with phosphate buffered saline (PBS) and then processed to obtain homogenate in $1 \mathrm{~mL}$ PBS and then stored at $-80^{\circ} \mathrm{C}$.

\section{Measurement of MMP-9 and E-cadherin}

After all samples were collected, the MMP9 and E-cadherin levels were assessed by the ELISA method based on the Cloud-Clone Corp protocol (MMP-9: SEA553Hu and E-cadherin: SEA017Hu). The results obtained from the ELISA test were then calculated based on the levels in total protein, then the MMP-9 units were $\mathrm{ng} / \mathrm{mg}$ and E-cadherin $\mathrm{pg} / \mathrm{mg}$.

\section{Ethical clearance}

This study received ethical permission from the Health Research Ethics Committee of the Faculty of Medicine, University of Indonesia No. 34/UN2.F1/ETIK/2017.

\section{Statistical analysis}

The distribution of abnormal data was tested based on the Kolmogorov-Smirnov test, The Mann-Whitney test to assess the comparative of clinical characteristics and levels of MMP-9 between the two groups. And independent-t test was used to compare levels of E-cadherin between EO-PE and LO-PE. A $p<0.05$ being considered as statistically significant. 


\section{RESULTS}

\section{Demographic information of the study population}

During the study we could only recruited 26 women in the EO-PE group and 38 in the LO$P E$ group. The demographic and clinical characteristics of 26 women with EO-PE and 38 women with LO-PE are summarized in Table 1. The median maternal age of women with EOPE [ 29.5 (range 21-42) years old] was not significantly different with median maternal age of women with LO-PE [30.5 (range (19-44) years old] $(\mathrm{p}=0.545)$ (Table 1). As expected, systolic blood pressure $(\mathrm{p}=0.607)$ and diastolic blood pressure $(p=0.658)$ was not significant different between EO-PE and LO-PE group. But semiquantitative proteinuria were significantly higher in the EO-PE group [3 (range $(0-4)$ ] compared to LO-PE group [1 range (0-3)] $(\mathrm{p}=0.000)$.

\section{Comparisons of MMP-9 and E-cadherin levels between EO-PE and LO-PE}

In Table 1, it was found that mean Ecadherin levels were lower in the EO-PE group $(125.94 \pm 54.22 \mathrm{pg} / \mathrm{mg})$ compared to LO-PE group $(157.95 \pm 54.12 \mathrm{pg} / \mathrm{mg})(\mathrm{p}=0.024)$. But there was not significant difference of median MMP9 levels between the two groups $(\mathrm{p}=0.376)$.

\section{DISCUSSION}

There was no significant difference of systolic and diastolic blood pressure between the
EO-PE and LO-PE. This result is different from the result obtained by Gomathy et al. ${ }^{(20)}$ that found diastolic blood pressure to be significantly higher in EO-PE than in LO-PE. This may be because there were patients in this study were already being treated with antihypertensives.

However, proteinuria was significantly higher at LO-PE compared to EO-PE group. Research conducted by Dong et al. ${ }^{(21)}$ showed proteinuria with a level of $0.3 \mathrm{~g} / \mathrm{L}$ was significantly higher in severe $P E$ than in mild PE. In our study, all EO- PE cases were PE cases with severe features. As it's already known, proteinuria was no longer included as a $P E$ according to the International Society of the Study of Hypertension in Pregnancy because it was found that multiple organ dysfunction affecting the kidneys and liver can appear without signs of protein and the amount of proteinuria cannot predict the severity of disease progression. ${ }^{(22)}$ However, although proteinuria is no longer recommended as a criterion for diagnosing $\mathrm{PE}$, in fact clinicians generally use proteinuria levels to inform clinical decisions regarding PE delivery cases. This is because increased levels of proteinuria worsen the development of $\mathrm{PE}$ and are associated with poor perinatal outcomes.

There was not significance difference levels of MMP-9 between EO-PE and LO-PE in this study. These results differed from the results of a study conducted by Timokhina et al. ${ }^{(23)}$ who found MMP-9 levels in EO-PE were lower than in LO-PE. These results showed the effect of MMP-9 on the spiral artery

Table 1. Comparison of demographic and clinical characteristics between EO and LO groups of preeclampsia patients

\begin{tabular}{lccc}
\hline \multicolumn{1}{c}{ Characteristics } & EO-PE $(\mathbf{n = 2 6})$ & LO-PE $(\mathbf{n}=\mathbf{3 8})$ & p value \\
\hline Maternal's age (years) & $29.5(21-42)$ & $30.5(19-44)$ & $0.545^{@}$ \\
Gestational age & $30(24-33)$ & $37(34-41)$ & $0.000^{@}$ \\
Systolic blood pressure $(\mathrm{mmHg})$ & $167.5(140-220)$ & $170(130-220)$ & $0.607^{@}$ \\
Diastolic blood pressure $(\mathrm{mmHg})$ & $100(88-150)$ & $110(90-140)$ & $0.658^{@}$ \\
Proteinuria (semiquantitative) & $3(0-4)$ & $1(0-3)$ & $0.000^{@}$ \\
MMP-9 (ng/mg) & $1.24(0.18-18.98)$ & $1.70(0.30-6.66)$ & $0.376^{@}$ \\
E-cadherin $(\mathrm{pg} / \mathrm{mg})$ & $125.94 \pm 54.22$ & $157.95 \pm 54.12$ & $0.024^{\$}$ \\
\hline
\end{tabular}

@ Mann Whitney test; ${ }^{\$}$ Independent t- test 
transformation process. Research conducted by Zhang et al. ${ }^{(12)}$ using immunohistochemical methods on placental samples found that there was a gradual decrease in patients with severe $\mathrm{PE}$ compared to mild PE and normal pregnancies.

As it's already known, in early gestation, an increase in MMP-9 is needed to regulate the angiogenesis process for the development of the foetus-maternal interface. While lower levels of MMP-9 in the maternal serum contribute to the abnormal development of blood vessels at the foetus-maternal interface in complicated pregnancies of severe PE. ${ }^{(8,24)}$ With the difference in this study related to MMP-9 levels, further research is needed to find changes in MMP-9 levels based on gestational age both at normal pregnancy and preeclampsia, so that it can be found more clearer regarding changes in MMP-9 levels.

Something different happened to Ecadherin. In this study it was found that Ecadherin was significantly lower EO-PE than LO-PE. Different results were found by $\mathrm{Li}$ et al. (7) who found no difference in E-cadherin expression between EO-PE and LO-PE. However, the expression of E-cadherin in LO$\mathrm{PE}$ was higher than in normal pregnancy, whereas the increase in E-cadherin expression did not correlate with the severity of preeclampsia. Li et al. ${ }^{(7)}$ concluded that Ecadherin increases cytotrophoblast proliferation and cytokeratin will be highly expressed in proliferative tissue and this causes impaired differentiation to become syncytio-trophoblast. Research conducted by Osman et al. ${ }^{(25)}$ in Sudan using immunohistochemical methods from placental samples found no difference in expression between severe PE and controls. Meanwhile, Du et al. ${ }^{(26)}$ found an increase in Ecadherin levels in PE patients compared to controls. The studies of Osman et al. ${ }^{(25)}$ and $\mathrm{Du}$ et al. ${ }^{(26)}$ did not examine the difference between EO-PE and LO-PE so it cannot be concluded whether there is a difference in levels between the two groups.
Limitations of this study were there was not enough sample size for EO-PE and the absence of normal gestational controls in comparable gestational age and the measurement of the levels did not follow the course of gestational age from early to term from the same patient. There is a need for a cohort study of gestational age to follow changes in levels in each pregnant woman so that a real change can be obtained. The results of this study indicate that we need to pay attention to the biomolecular changes that occur in EO-PE so that it can reduce the risk of premature birth.

\section{CONCLUSION}

This study demonstrated that E-cadherin expression levels was significantly lower in EO$\mathrm{PE}$. It shows that lower levels of e-cadherin can lead to early delivery in preeclampsia patients. Further studies are needed using normal pregnancy and preeclampsia patients in all gestational age to examine the levels of MMP9 and E-cadherin and its relationship.

\section{CONFLICT OF INTEREST}

There is no conflict of interest

\section{ACKNOWLEDGMENT}

The authors thank the staff of the Cipto Mangunkusumo Hospital, Budi Kemuliaan Hospital, and the Integrated Laboratory of Medical faculty of Universitas Indonesia for their assistance and support.

\section{CONTRIBUTORS}

TDS contributed to collecting samples, draft manuscripts, collecting data, and analyse data. HW contributed to analyse data. IKL contributed to manuscript finalization. ARP contributed to the research concept. A contributed to the research design and manuscript revision. All authors have read and agreed to the final manuscript. 


\section{REFFERENCES}

1. The Preeclampsia Foundation. New guidelines in preeclampsia diagnosis and care Include revised definition of preeclampsia. Melbourne, FL USA;2013.

2. Eiland E, Nzerue C, Faulkner M. Preeclampsia. J Pregnancy 2012;2012. doi: 10.1155/2012/586578.

3. Ardian M, Dachlan E. The characteristic of Indonesia's pre-eclampsia: From obstetric intensive care with ventilator until epidemiologic and its molecular biology profile of pulmonary edema in severe pre-eclampsia. Pregnancy Hypertens 2014;4:245. doi: 10.1016/ j.preghy.2014.04.018.

4. Savaj S, Vaziri N. An overview of recent advances in pathogenesis and diagnosis of preeclampsia. Iran J Kidney Dis 2012;6:334-8. doi: 10.1073/pnas. . 1816150116.

5. Sheridan MA, Yang Y, Jain A, et al. Early onset preeclampsia in a model for human placental trophoblast. PNAS. 2019;116:4336-45. doi: 10.1073/pnas.. 1816150116.

6. Meeme A, Buga GA, Mammen M, Namugowa AV. Angiogenic imbalance as a contributor to the pathophysiology of preeclampsia among black African women. J Matern Fetal Neonatal Med 2017;30:1335-41. doi: 10.1080/14767058.2016. 1212832.

7. Li X, Dong X, Xue Y, Li C, Gou W, Chen Q. Increased expression levels of E-cadherin, cytokeratin 18 and 19 observed in preeclampsia were not correlated with disease severity. Placenta 2014;35:625-31. doi: 10.1016/j.placenta.2014. 04.010 .

8. Laskowska M. Altered maternal serum matrix metalloproteinases MMP-2, MMP-3, MMP-9, and MMP-13 in severe early-and late-onset preeclampsia. Bio Med Res Int 2017;2017. https:/ /doi.org/10.1155/2017/6432426.

9. Maître JL, Heisenberg CP. Three functions of cadherins in cell adhesion. Curr Biol 2013;23:R626R33. doi: 10.106/j.cub.2013.06.019.

10. Howard S, Deroo T, Fujita Y, Itasaki N. A positive role of cadherin in $\mathrm{Wnt} / \beta$-catenin signalling during epithelial-mesenchymal transition. PLoS One 2011;6:e23899. doi: 10.1371/journal.pone. 0023899.

11. Zhao HB, Wang C, Li RX, et al. E-cadherin, as a negative regulator of invasive behavior of human trophoblast cells, is down-regulated by cyclosporin A via epidermal growth factor/ extracellular signal-regulated protein kinase signaling pathway. Biol Reprod 2010 ;83:370-6. doi: 10.1095/biolreprod.110.083402.
12. Zhang Y, Li P, Guo Y, Liu X, Zhang Y. MMP-9 and TIMP-1 in placenta of hypertensive disorder complicating pregnancy. Exp Ther Med 2019;18:637-41. doi: 10.3892/etm.2019.7591.

13. Yabluchanskiy A, Ma Y, Iyer RP, Hall ME, Lindsey ML. Matrix metalloproteinase-9: many shades of function in cardiovascular disease. Physiology 2013;28:391-403. doi: 10.1152/physiol.00029.2013.

14. Han S, Han L, Sun H, et al. Krüppel like factor expression and correlation with FAK, MMP 9 and E cadherin expression in hepatocellular carcinoma. Mol Med Report 2013;8:81-8. doi: 10.3892/mmr. 2013.1471.

15. Sosa EY, Flores-Pliego A, Espejel-Nuñez A, et al. New insights into the role of matrix metalloproteinases in preeclampsia. Int J Mol Sci 2017;18:1448. doi: 10.3390/ijms18071448.

16. Palei AC, Sandrim VC, Amaral LM, et al. Association between matrix metalloproteinase (MMP)-2 polymorphisms and MMP-2 levels in hypertensive disorders of pregnancy. Exp Mol Pathol 2012;92:217-21. doi: 10.1016/j.yexmp. 2012.01.008.

17. Roberts JM, Rich-Edwards JW, McElrath TF, Garmire L, Myatt L for the Global Pregnancy Collaboration. Subtypes of preeclampsia: recognition and determining clinical usefulness. Hypertension 2021;77:1430-41. DOI: 10.1161/ HYPERTENSIONAHA.120.14781.

18. Meng H, Qi M, Liu Y, Xu L, Li Q. Research on correlation between MMP-9 and early-onset preeclampsia. Int J Clin Exp Med 2016;9:17442-8.

19. Li XL, Dong X, Xue Y, Li CF, Gou WL, Chen Q. Increased expression levels of E-cadherin, observed in preeclampsia were not correlated with disease severity. Placenta 2014;35:625-31. doi: 10.1016/j.placenta.2014.04.010.

20. Gomathy E, Akurati L, Radhika K. Early onset and late onset preeclampsia-maternal and perinatal outcomes in a rural teritiary health center. Int J Reprod Contracept Obstet Gynecol 2018;7:22669. https://dx.doi.org/10.18203/2320-1770. ijrcog20182333.

21. Dong X, Gou W, Li C, et al. Proteinuria in preeclampsia: Not essential to diagnosis but related to disease severity and fetal outcomes. Pregnancy Hypertens 2017;8:60-4. doi: 10.1016/ j.preghy.2017.03.005.

22. Brown MA, Magee LA, Kenny LC, et al. on behalf of the International Society for the Study of Hypertension in Pregnancy (ISSHP). Hypertensive disorders of pregnancy : ISSHP classification, diagnosis, and management recommendations for international practice. Hypertension 2018;72:24-43. DOI: 10.1161/ HYPERTENSIONAHA.117.10803. 
23. Timokhina E, Strizhakov A, Ibragimova S, et al. Matrix metalloproteinases MMP-2 and MMP-9 occupy a new role in severe preeclampsia. J Pregnancy 2020;2020. https://doi.org/10.1155/ 2020/8369645.

24. Laskowska M. Maternal serum matrix metalloproteinase 9 in pregnancies complicated by severe preeclampsia and/or intrauterine fetal growth restriction. MOJ Women's Health 2017;4:127-31. doi: 10.15406/mojwh.2017.04.00099.
25. Osman SE, Salih M, Ahmed EM, Mohammed AA Adam I. Expression of E-cadherin, N-cadherin, and cytokeratin 18 and 19 in placentas of women with severe preeclampsia. Open Access Macedonian J Med Sci 2020;8:485-9. doi: 10.3889/ oamjms.2020.4004.

26. Du L, Kuang L, He F, Tang W, Sun W, Chen D. Mesenchymal-to-epithelial transition in the placental tissues of patients with preeclampsia. Hypertens Res 2017;40:67-72. doi: 10.1038/ hr.2016.97. 